\title{
The Algebraic Structure of Quantity Calculus II: Dimensional Analysis and Differential and Integral Calculus
}

\author{
Álvaro P. Raposo ${ }^{1}$ \\ ${ }^{1}$ Department of Applied Mathematics, Universidad Politécnica de Madrid, Av. Juan de Herrera, 6, 28040, Madrid, Spain, \\ alvaro.p.raposo@upm.es
}

In a previous paper, the author has introduced and studied a new algebraic structure which accurately describes the algebra underlying quantity calculus. The present paper is a continuation of that one, which extends the purely algebraic study by adding two more ingredients: an order structure and a topology. The goal is to give a solid justification of dimensional analysis and differential and integral calculus with quantities.

Keywords: Quantity calculus, algebraic structure, dimensional analysis, pi-theorem.

\section{INTRODUCTION}

In a previous paper [1] the author has produced a new algebraic structure, axiomatically defined, called algebraic space of quantities, which resembles accurately the algebra structure of quantity calculus. In that paper there is a review of past attemps of giving a mathematical framework to quantity calculus and of the motivation to introduce yet another, different, algebraic structure which is now summarized here. Despite in the standard approach to quantity calculus (e.g. the International Vocabulary of Metrology (VIM) [2]) the calculus focuses on quantities and dimensions as central concepts, the mathematical approach to the algebraic structure underlying quantity calculus is based on units as the main concept. In the recent analysis of Kitano [3], for instance, or the first papers on this model, [4], [5], [6], the model is set up starting from a set of base units $\left(u_{1}, \ldots, u_{n}\right)$ and expresses any quantity $q$ in terms of them as

$$
q=\alpha u_{1}^{r_{1}} \cdots u_{n}^{r_{n}}
$$

where $\alpha, r_{1}, \ldots, r_{n}$ are real numbers. In this equation $\alpha$ is the numerical value of $q$ with respect to this system of units, while $u_{1}^{r_{1}} \cdots u_{n}^{r_{n}}$ is the unit in which $q$ is measured. The set of all units, that is $\left\{u_{1}^{r_{1}} \cdots u_{n}^{r_{n}}: r_{i} \in \mathbb{R}, i=1, \ldots, n\right\}$ has the structure of a vector space over $\mathbb{R}$, written in multiplicative form. The set of quantities, thus, adopts the form of a family of rays, each identified and spanned by a unit as in (1) by letting $\alpha$ run through the reals. The rays coincide in a point, the zero of the algebraic structure. The numbers $\left(r_{1}, \ldots, r_{n}\right)$ are referred to as the dimensions of the quantity $q$.

This algebraic structure has served its main purpose: giving a proof of Buckingham's Pi Theorem. However, it has DOI: 10.2478/msr-2019-0012 two drawbacks which show it not to be an accurate description of the actual algebraic properties of quantity calculus. First, it is based on the concept of unit, as all the structure is built upon a starting set of base units. In fact, quantities are introduced as objects of the form a number times a unit (just as Maxwell did over 100 years ago [7]), thus identifying the concepts of quantity and quantity value, which are well differentiated by the VIM. This is quite unsatisfactory, for this approach blurs the distinction between the system of quantities (such as the ISQ) and the system of units (such as the SI). This situation has been recognized by several authors in previous years (see de Boer [8] for an excellent review and discussion, [9] for a recent one) who have claimed, instead, for an algebraic structure defined axiomatically and based on the concept of quantity and of dimension rather than on the concept of unit, which should be secondary.

The second drawback is the use of real exponents in (1). They are not necessary. It is a remarkable fact that the laws of Physics involve dimensionful quantities (in contrast with dimensionless quantities) only through three operations: product of quantities, product by a scalar, and addition of quantities of the same kind. Therefore, only integer exponents are found in physical equations. Some simple examples seem to prove the previous statement wrong, for instance, the velocity $v$ of a body of mass $m$ for which the kinetic energy $T$ is known is given by

$$
v=\sqrt{\frac{2 T}{m}},
$$

so the dimensionful quantity $T / m$ has an exponent 0.5 . However, the dimension of $T / m$ is already a squared one: $[T / m]=$ 
$\left(\mathrm{LT}^{-1}\right)^{2}$, so the use of the exponent 0.5 is only, say, accidental. The period $t$ of a pendulum given its length $l$ and the acceleration of free fall, $g$, provides a similar example, for it is given by

$$
t=2 \pi \sqrt{\frac{l}{g}},
$$

but, again, the quantity $l / g$ has a squared dimension, $[l / g]=$ $\mathrm{T}^{2}$, so the exponent 0.5 does not provide a dimension with fractional exponent. A more critical example is a system of units in which volume is taken as a base quantity and, e.g. the liter as the corresponding base unit. In that case we should admit fractional exponents for the derived units for length, $(\text { liter })^{1 / 3}$, or surface area, (liter $)^{2 / 3}$. Nevertheless, experience in measurement science has shown, so far, that a suitable choice of base quantities is possible which avoids such fractional exponents to define units, and this is the case this paper wants to support from the mathematical point of view. With this goal in mind, the allowance of real exponents is unnecessarily oversized. It works, of course, since real numbers contain integer numbers, but it is not the algebraic structure which best fits the quantity calculus.

The paper [1] addresses these two issues and gives a way out by defining, in axiomatic form, a new algebraic structure which resembles, more accurately, the calculus with quantities (i.e., only integer exponents allowed) and is completely focused on quantities and dimensions. It starts with the definition of the group of dimensions $\mathscr{D}$ as a finitely generated free Abelian group. Its elements are denoted A, B... and are referred to as dimensions. The identity of the group is denoted $1 \mathscr{D}$.

In the quantity calculus real numbers play a prominent role as the measurement of a quantity is nothing but its comparison with the unit of its kind, and the result is a real number. This number and the unit make the quantity value. In the definition of the algebraic structure, the role of real numbers is taken by an abstract field $F$. Nevertheless, this abstract field is going to get more specific along the sections of this paper, first as an ordered field in section 3 , then as the real numbers in sections 4 and 5 . With these elements, a summary of the definition given in [1] is as follows. A space of quantities $Q$ with group of dimensions $\mathscr{D}$ over the field $F$ is a set $Q$, whose elements are called quantities, together with a surjective map $\operatorname{dim}: Q \rightarrow \mathscr{D}$ and three operations. For each dimension A, the set $\operatorname{dim}^{-1} A$ is called a fiber, and the first two operations are defined within each fiber: addition of quantities and product by scalars in the field $F$. With them, the fiber aquires the structure of a one-dimensional vector space over $F$. In addition, the whole set $Q$ has a product, the third operation, with respect to which it is an Abelian monoid; the neutral element is $1_{Q}$. As the three operations and the map dim are related by, first, dim is a homomorphism with respect to the product in $Q$, second, distribution of the product over the addition in each fiber and, third, association of the product of quantities and product by a scalar. The structure is referred to as an algebraic fiber bundle and denoted by the symbol $Q \stackrel{\operatorname{dim}}{\longrightarrow} \mathscr{D}$.

A key and distinctive feature of this structure is that each fiber has its own zero element. The fiber $\operatorname{dim}^{-1} 1_{\mathscr{D}}$ is called the set of dimensionless quantities or, more appropriately, quantities of dimension one. This fiber is, in addition to the structure of vector space, an algebra over $F$ and, in fact, is naturally isomorphic with $F$ by means of the map $F \rightarrow$ $\operatorname{dim}^{-1} 1_{\mathscr{D}}: \alpha \mapsto \alpha 1_{Q}$, so we identify both whenever needed.

Notice that the structure of the fibers as one dimensional vector spaces cannot be the ultimate model of quantity calculus, for it does not fit properly to quantized quantities. If a quantity is quantized, the corresponding fiber should not be the whole vector space, but a discrete subset of it. However, this issue will not be dealt with in this paper.

A system of units is a section of the fiber bundle, that is, a map $\sigma: \mathscr{D} \rightarrow Q$ such that $\operatorname{dim} \circ \sigma=\mathrm{id}_{\mathscr{D}}$. The system is coherent if $\sigma$ is a group homomorphism, and it is nonzero if $\sigma(\mathrm{A})$ is not a zero for any dimension A. In [1] the conditions for its existence are established.

In this paper we only consider spaces of quantities free of zero divisors. Two advantages from such a choice are: (i) every nonzero quantity has an inverse and (ii) there exists a nonzero coherent system of units. So we also assume without further notice that such a system is always available and all systems of units considered are nonzero and coherent.

When a coherent nonzero system of units $\sigma$ is defined in a space of quantities then we have the map $v: Q \rightarrow F$ (where we identify the fiber of dimensionless quantities with $F$ ) defined by

$$
v(q)=q \sigma(\operatorname{dim}(q))^{-1},
$$

which assigns to a quantity $q$ its numerical value with respect to the system of units $\sigma$; it is a homomorphism. Notice that the composition $\sigma \circ \operatorname{dim}: Q \rightarrow Q$ gives the unit in the fiber of each quantity. Then, a quantity $q$ is written in terms of a system of units as

$$
q=v(q) \sigma \circ \operatorname{dim}(q)
$$

With the units at hand paper [1] explores the way in which quantities and the operations among them are expressed in terms of the units. Ways to create new spaces from old ones (subspace, tensor product, quotient space) are also explored and, finally, the tool for comparison is defined, the homomorphism of spaces of quantities, and utilized to give a characterization theorem of these structures. Finally, it is demonstrated that any such structure, given a system of units, is isomorphic with the projection fiber bundle $F \times \mathscr{D} \stackrel{\operatorname{dim}}{\longrightarrow} \mathscr{D}$.

As can be seen, paper [1] merely defines and develops the algebraic tools. Now it is the time to apply them to give a sound justification to the two main uses of quantity calculus beyond the algebraic operations: dimensional analysis and differential and integral calculus. However, before addressing these two questions, a bit more of structure is needed over the algebraic base: an order structure. And also a tool that appears once and again along the paper: changes of units. Therefore, the layout is the following: changes of units are treated in section 2, and the order structure in section 3 . Section 4 deals with dimensional analysis and states and proves 
the Pi-theorem in this context. Finally, in section 5, the basis of differential calculus and of integral calculus with quantities is established.

\section{Change OF Units}

In this section we study the way to change from a system of units to another, that is, a change of scale. Consider two systems of units $\sigma_{1}$ and $\sigma_{2}$. The quantity $q$ can be expressed in terms of both of them according to (5) as $q=v_{1}(q) \sigma_{1}(\operatorname{dim}(q))=v_{2}(q) \sigma_{2}(\operatorname{dim}(q))$. The quantity $\sigma_{2}(\operatorname{dim}(q))$ is not zero, so it has an inverse and we can solve for $v_{2}(q)$ as

$$
v_{2}(q)=\sigma_{1}(\operatorname{dim}(q)) \sigma_{2}(\operatorname{dim}(q))^{-1} v_{1}(q),
$$

which is the conversion formula between the numerical values with respect to both systems of units. The number $\sigma_{1}(\operatorname{dim}(q)) \sigma_{2}(\operatorname{dim}(q))^{-1}$ is the conversion factor, or scale factor, for the fiber of $q$. Notice that this equation seems different from the usual conversion factor formula, which would read $\sigma_{1}=\left(v_{2} / v_{1}\right) \sigma_{2}$; however, this form is not useful for mathematical purposes because $v_{1}$ may be zero, and thus division is not allowed. On the other hand, $\sigma_{2}$ is always nonzero by definition, so the division is safe.

The association of the scale factor with each fiber (equivalently, with each dimension in $\mathscr{D}$ ) is a group homomorphism, and the converse is also true:

Proposition 2.1. Given two systems of units $\sigma_{1}$ and $\sigma_{2}$, the scale factor map $\chi: \mathscr{D} \rightarrow F^{*}$ defined by $\chi(\mathrm{A})=$ $\sigma_{1}(\mathrm{~A}) \sigma_{2}(A)^{-1}$ is a group homomorphism. Conversely, given a group homomorphism $\chi: \mathscr{D} \rightarrow F^{*}$ and a system of units $\sigma$, the map $\tilde{\sigma}: \mathscr{D} \rightarrow Q$ defined by $\tilde{\sigma}(\mathrm{A})=\chi(\mathrm{A}) \sigma(\mathrm{A})$ is a system of units.

Proof. For the direct statement, let $\mathrm{A}$ and $\mathrm{B}$ be dimensions in $\mathscr{D}$. Then

$$
\begin{aligned}
\chi(\mathrm{AB})=\sigma_{1}(\mathrm{AB}) \sigma_{2}(\mathrm{AB})^{-1}= \\
\quad \sigma_{1}(\mathrm{~A}) \sigma_{1}(\mathrm{~B})\left(\sigma_{2}(\mathrm{~A}) \sigma_{2}(\mathrm{~B})\right)^{-1}= \\
\quad \sigma_{1}(\mathrm{~A}) \sigma_{2}(\mathrm{~A})^{-1} \sigma_{1}(\mathrm{~B}) \sigma_{2}(\mathrm{~B})^{-1}=\chi(\mathrm{A}) \chi(B),
\end{aligned}
$$

because both, $\sigma_{1}$ and $\sigma_{2}$ are group homomorphisms and all the products are commutative.

For the converse we have to prove $\tilde{\sigma}$ is (i) a section of $Q$, (ii) nonzero, and (iii) coherent.

i. Let $\mathrm{A}$ be a dimension. Then

$$
\begin{aligned}
\operatorname{dim} \circ \tilde{\sigma}(\mathrm{A})= & \operatorname{dim}(\chi(\mathrm{A}) \sigma(\mathrm{A}))= \\
& \operatorname{dim}(\chi(\mathrm{A})) \operatorname{dim}(\sigma(\mathrm{A}))=1_{\mathscr{D}} \mathrm{A}=\mathrm{A},
\end{aligned}
$$

where we have identified the number $\chi(\mathrm{A})$ with a dimensionless quantity. Since $A$ is arbitrary, $\operatorname{dim} \circ \tilde{\sigma}=\mathrm{id}_{\mathscr{D}}$.

ii. Since $\chi(\mathscr{D}) \subset F^{*}$ and $\sigma$ is nonzero, clearly $\tilde{\sigma}$ is nonzero as well. iii. Let $A$ and $B$ be dimensions. Then

$$
\begin{aligned}
& \tilde{\sigma}(\mathrm{AB})=\chi(\mathrm{AB}) \sigma(\mathrm{AB})= \\
& \chi(\mathrm{A}) \chi(\mathrm{B}) \sigma(\mathrm{A}) \sigma(\mathrm{B})=\tilde{\sigma}(\mathrm{A}) \tilde{\sigma}(\mathrm{B}),
\end{aligned}
$$

because both, $\chi$ and $\sigma$ are group homomorphisms and the commutativity of the products.

Let us go a step further by considering the set $\mathscr{G}$ of all scale factor maps $\chi: \mathscr{D} \rightarrow F^{*}$. Let $\chi, \xi$ be two such maps; we can define a product in $\mathscr{G}$ componentwise, that is, for a dimension $\mathrm{A},(\chi \xi)(\mathrm{A})=\chi(\mathrm{A}) \xi(\mathrm{A})$. With this product the set $\mathscr{G}$ is an Abelian group, the identity element is the map $\mathrm{A} \mapsto 1$, i.e. with all scale factors 1 , and the inverse element of $\chi$ is $\chi^{-1}(\mathrm{~A})=\chi(\mathrm{A})^{-1}$. We refer to this group later in section 3 .

\section{ORDER STRUCTURE IN A SPACE OF QUANTITIES}

We all agree that $3 \mathrm{~kg}$ is a greater mass than $2 \mathrm{~kg}$, and that $3 \mathrm{~kg}$ and $5 \mathrm{~m} / \mathrm{s}$ are not comparable. These examples show that the addition of an order relation to the algebraic structure of a space of quantities must be studied in order to complete the mathematical model of quantity calculus. And it also warns us that the order needs not be complete, but a partial order.

The order we are about to define is borrowed from an order in the base field $F$ so, in this section, $F$ is assumed to be an ordered field, such as $\mathbb{Q}$ or $\mathbb{R}$, and the order relation in the field is denoted by $<$.

The first step is to define what we understand by an order relation compatible with the algebraic structure of a space of quantities.

Definition 3.1. An ordered space of quantities $Q \stackrel{\operatorname{dim}}{\longrightarrow} \mathscr{D}$ over the ordered field $F$ is a space of quantities with an order relation $\prec$ in $Q$ such that:

$i$. In each fiber there is a quantity greater than the zero of the fiber.

ii. Let $a_{1}, a_{2}, a_{3}$ be quantities in the same fiber. If $a_{1} \prec a_{2}$ then $a_{1}+a_{3} \prec a_{2}+a_{3}$.

iii. Let $a$ and $b$ be quantities and $\gamma$ a scalar number in $F$. If $a \prec b$ and $\gamma>0$, then $\gamma a \prec \gamma b$ and, if $a \prec b$ and $\gamma<0$, then $\gamma b \prec \gamma a$.

iv. Let $a, b$ and $c$ be quantities, $\operatorname{dim}(c)=$ C. If $a \prec b$ and $0_{\mathrm{C}} \prec c$ then $a c \prec b c$.

Remarks: The first item of the definition is needed because, as noticed above, the order relation needs not be a total order, so we want at least to be able to make a comparison inside each fiber. A quantity that is greater than the zero of its fiber is called positive. The remaining three items are the compatibility conditions with each of the three operations in $Q$. The second part of item (iii) is not needed in a fiber, for it can be deduced from (ii), but that is not the case when it applies to the comparison of quantities of different dimensions, since addition among them is not defined.

A number of immediate consequences follows from the previous definition. 
Proposition 3.2. Let $Q \stackrel{\mathrm{dim}}{\longrightarrow} \mathscr{D}$ be an ordered space of quantities over the field $F$. Then

$i$. The fiber of dimensionless quantities is naturally order isomorphic with $F$.

ii. Each fiber is order isomorphic with $F$.

iii. Zeros of different fibers are not comparable.

$i v$. The rule of signs applies to the product of quantities

v. If a quantity is positive, its inverse is also positive (in its corresponding fiber).

PROOF. i. Remember that the natural map between the field $F$ and the fiber of dimensionless quantities $F \rightarrow$ $\operatorname{dim}^{-1}\left(1_{\mathscr{D}}\right): \alpha \mapsto \alpha 1_{Q}$ is an isomorphism of algebras, so we only need to prove it preserves the order. By (i) in definition 3.1, there is $\alpha \in F$ such that $0_{1_{\overparen{D}}} \prec \alpha 1_{Q}$, so $\alpha \neq 0$. Assume $\alpha<0$ in $F$, then $-\alpha^{-1}>0$ and so, by (iii) in the same definition, $0_{1_{\mathscr{D}}} \prec-\alpha^{-1} \alpha 1_{Q}=-1_{Q}$, therefore $1_{Q} \prec 0_{1}$. Multiply the later by the positive quantity $-1_{Q}$ and get $-1_{Q} \prec 0_{1_{\mathscr{g}}}$, a contradiction with the antisymmetry of the order relation. If, on the contrary, $\alpha>0$ then so is $\alpha^{-1}$ and, by the same argument, we get $0_{1_{\mathscr{D}}} \prec 1_{Q}$.

ii. Let $\mathrm{A}$ be a dimension in $\mathscr{D}$ and $a$ a positive quantity in the fiber of dimension A. The map $F \rightarrow \operatorname{dim}^{-1}(\mathrm{~A}): \alpha \mapsto$ $\alpha a$ is an order isomorphism (in fact, it is also a linear isomorphism) for, if $\alpha_{1}<\alpha_{2}$ in $F$, then $0<\alpha_{2}-\alpha_{1}$ so we can multiply in $0_{\mathrm{A}} \prec a$ and get $0_{\mathrm{A}} \prec\left(\alpha_{2}-\alpha_{1}\right) a$. Now, by (ii) in the definition 3.1, we conclude $\alpha_{1} a \prec$ $\alpha_{2} a$, so the linear isomorphism preserves the order.

Notice this item is different from the first one because the isomorphism is not canonical; it depends on the arbitrarily chosen quantity $a$. Now we can denote as positive all quantities greater than zero in a fiber, and as negative their negatives, which are less than zero. And we can also define the absolute value of a quantity $q$ as $|q|=q$ if $q$ is positive or zero, or $|q|=-q$ in case it is negative.

iii. Let $A$ and $B$ be different dimensions. We already know $0_{\mathrm{A}} \neq 0_{\mathrm{B}}$ because $\operatorname{dim}\left(0_{\mathrm{A}}\right)=\mathrm{A} \neq \mathrm{B}=\operatorname{dim}\left(0_{\mathrm{B}}\right)$. Assume $0_{A} \prec 0_{B}$. Then, after multiplication by -1 , by (iii) in the definition, we get $0_{B} \prec 0_{A}$, a contradiction with the antisymmetry of the order relation..

iv. Let $a$ and $b$ be positive quantities of dimensions $\mathrm{A}$ and $\mathrm{B}$ respectively. Then, by (iv) in the definition, $0_{\mathrm{AB}} \prec a b$. Now, if both quantities were negative, then by (iii) in the same definition, $-a$ and $-b$ are positive, so $0_{\mathrm{AB}} \prec$ $(-a)(-b)=a b$. Similarly, in the cases of different signs we get $a b \prec 0_{\mathrm{AB}}$.

v. Let $a$ be a positive quantity with $\operatorname{dim}(a)=\mathrm{A}$. Since it is not zero, it is invertible and $a^{-1}$ is not zero as well so, by the second item of this proposition, it is greater or less than $0_{A^{-1}}$. In the later case, multiplication by the positive quantity $a$ yields $1_{Q} \prec 0_{1_{\mathscr{D}}}$, a contradiction.
In the following considerations there is no need to distinguish the symbols $\prec$ (order in $Q$ ) from $<$ (order in $F$ ) for we have seen they share the same properties, so we only use the more familiar $<$ for both. Nevertheless, the context always makes it clear which is the case.

In the definition of an ordered space of quantities there is nothing to prevent a comparison of quantities of different dimensions. We now turn to study this issue to see that, although the algebraic and order structures can hold such a comparison, the consequences are far from what it is desirable for a quantity calculus. As we can see in the following paragraphs, all the discussion can be reduced to the fact that zeros of different fibers are not comparable (third item of the previous proposition).

Let us assume there are quantities $a$ and $b$, with $\operatorname{dim}(a)=$ $\mathrm{A} \neq \mathrm{B}=\operatorname{dim}(b)$ such that $a<b$. We can assume $0_{\mathrm{B}}<b$, otherwise choose a quantity $b^{\prime}$ greater than $0_{\mathrm{B}}$ and, by transitivity of the order, $a<b<b^{\prime}$, so $a<b^{\prime}$ as desired.

Now, regarding $a$, consider the two possibilities either $0_{\mathrm{A}}<$ $a$ or $a<0_{\mathrm{A}}$. In the first case, again by the transitivity of the order relation, we get

$$
0_{\mathrm{A}}<b .
$$

Define the sets $\mathscr{B}^{ \pm}=\left\{x \in \operatorname{dim}^{-1}(\mathrm{~B}): 0_{\mathrm{A}} \lessgtr x\right\}$. It is easy to see that any $b^{\prime}$ such that $0_{\mathrm{B}}<b^{\prime}$ is in $\mathscr{B}^{+}$, because $b^{\prime}=\beta b$ for some number $\beta>0$ so, after multiplication in (7), we get $0_{\mathrm{A}}<\beta b=b^{\prime}$. Also, multiplication of (7) by -1 gives $-b<0_{\mathrm{A}}$ and then, by a similar argument, if $b^{\prime}<0_{\mathrm{B}}$ then $b^{\prime}$ is in $\mathscr{B}^{-}$. In other words, for any $b^{\prime}$ such that $0_{\mathrm{B}}<b^{\prime}$ we have $-b^{\prime}<0_{\mathrm{A}}<b^{\prime}$.

But this does not necessarily mean that there are quantities of dimension $B$ as close to $0_{A}$ as desired, for it may be the case that $a^{\prime}<b^{\prime}$ for any quantity $a^{\prime}$ with dimension $\mathrm{A}$ and any positive quantity $b^{\prime}$. In this later case, the whole fiber $\operatorname{dim}^{-1}(\mathrm{~A})$ lies above negative elements of $\operatorname{dim}^{-1}(B)$ and below positive elements of $\mathrm{dim}^{-1}(B)$. However, while $0_{A}$ is comparable with all elements of the fiber of $B$ (except its zero), $0_{B}$ is comparable with no element at all of the fiber of $A$.

To explore another scenario, assume in addition that there is $a^{\prime}$ in $\operatorname{dim}^{-1}(\mathrm{~A})$ which is greater than $b$, so we have $a<$ $b<a^{\prime}$. By transitivity we get $0_{\mathrm{B}}<b<a^{\prime}$ so the same argument can be utilized here and define the sets $\mathscr{A}^{ \pm}$of elements in $\operatorname{dim}^{-1}(\mathrm{~B})$ greater or less, respectively, than $0_{\mathrm{B}}$. Moreover, since we also have $0_{\mathrm{A}}<b<a^{\prime}$, let $a^{\prime \prime}$ be any positive quantity in $\operatorname{dim}^{-1}(\mathrm{~A})$, then $a^{\prime \prime}=\alpha a^{\prime}$ for some number $\alpha>0$ so, after multiplication by $\alpha, 0_{\mathrm{A}}<\alpha b<\alpha a^{\prime}=a^{\prime \prime}$, i.e. we can find an element in the fiber of $B$ between $0_{A}$ and any positive element in the fiber of $A$. The same applies to negative elements. Therefore, in this case we can say that there are elements in $\operatorname{dim}^{-1}(B)$ as close to $0_{A}$ as we desire. Even more, the closer an element in the fiber $\operatorname{dim}^{-1}(B)$ is to $0_{B}$, the closer it is to $0_{A}$. However, $0_{A}$ and $0_{B}$ remain uncomparable.

A similar discussion can be made in the case $a<0_{\mathrm{A}}, 0_{\mathrm{B}}<b$ and $a<b$, leading to similar conclusions.

Since the scenarios depicted by such ordinations of quantities of different dimensions are not in accordance with the 
actual use of quantity calculus in Physics, in the remaining we only consider order structures in which quantities of different dimensions are not comparable. Notice that, by item (ii) of the proposition 3.2, in this case the order is characterized by defining an element in each fiber as positive, i.e. giving an orientation to each fiber. But this choice is bound with algebraic restrictions. For instance, either if we choose a quantity $a$, with dimension $\mathrm{A}$, to be positive or we choose $-a$ to be positive, in both cases $a^{2}$ is a positive quantity in the fiber of $A^{2}$. Thus, we are free to choose an orientation only for the fibers of a basis in the group of dimensions. This fact gives full meaning to the following definition.

Definition 3.3. An ordered space of quantities $Q \stackrel{\operatorname{dim}}{\longrightarrow} \mathscr{D}$ is oriented by a system of units $\sigma$ if, for any dimension $\mathrm{A}, 0_{\mathrm{A}}<$ $\sigma(\mathrm{A})$, that is, if all units are positive in their respective fibers.

All the previous definitions can be summarized by saying that the bundle's fibers are 1-dimensional positively oriented linearly ordered vector spaces.

If a space of quantities is oriented by a system of units then the order relation is translated to the numerical values of the quantities with respect to the system of units, which is the usual practice, as seen in the following proposition.

Proposition 3.4. Let $Q \stackrel{\operatorname{dim}}{\longrightarrow} \mathscr{D}$ be oriented by the system of units $\sigma$ and let $a_{1}$ and $a_{2}$ be quantities with $\operatorname{dim}\left(a_{1}\right)=$ $\operatorname{dim}\left(a_{2}\right)=$ A. Then $a_{1}<a_{2}$ if and only if $v\left(a_{1}\right)<v\left(a_{2}\right)$.

PROOF. For the only if part, let $a_{1}<a_{2}$ and remember that these quantities are written in terms of the system of units as $a_{i}=v\left(a_{i}\right) \sigma(\mathrm{A}), i=1,2$, so we have $v\left(a_{1}\right) \sigma(\mathrm{A})<$ $v\left(a_{2}\right) \sigma(\mathrm{A})$. Since $0_{\mathrm{A}}<\sigma(\mathrm{A})$ by hypothesis then, by proposition $3.2, \sigma(\mathrm{A})^{-1}$ is positive, so we can multiply and get $v\left(a_{1}\right)<v\left(a_{2}\right)$.

Conversely, if $v\left(a_{1}\right)<v\left(a_{2}\right)$, just multiply by the positive quantity $\sigma(\mathrm{A})$ to get $a_{1}<a_{2}$.

In fact, this is the common way to define an order in a space of quantities, by setting units and regarding them as positive. It is easy to check that an order defined as in the previous proposition satisfies the conditions of definition 3.1 of an ordered space of quantities. In the following we write "an order defined by $\sigma$ " or "an order with respect to $\sigma$ " as equivalents to "an ordered space oriented by $\sigma$ ".

We have seen that a system of units fixes an order structure in a space of quantities. Since systems of units are arbitrarily chosen (from the algebraic viewpoint), we want to explore to what extent this arbitrariness extends to the allowed order structures in a space of quantities. To that end we make use of the group structure of $\mathscr{G}$, the group of scale factor maps, that is, changes of units. First remember that the ordered field $F$ can be split as the disjoint union $F=F^{+} \cup\{0\} \cup F^{-}$, where $F^{+}$is the so called positive cone of $F$, that is, the elements $\alpha>0$, and $F^{-}=-F^{+}$. If we look at the group structure of $F^{*}=F^{+} \cup F^{-}$, we easily discover that $F^{+}$is a subgroup and the quotient $F^{*} / F^{+} \cong C_{2}$, the cyclic group of order 2 , which is the group theoretical formulation of the rule of signs.

Let $\sigma$ and $\tilde{\sigma}$ be two systems of units. Then there is a scale factor map $\chi: \mathscr{D} \rightarrow F^{*}$ which relates them in the form
$\tilde{\sigma}(\mathrm{A})=\chi(\mathrm{A}) \sigma(\mathrm{A})$ for $\mathrm{A} \in \mathscr{D}$. The orders defined by $\sigma$ and $\tilde{\sigma}$ in each fiber, denoted $<$ and $\tilde{<}$, respectively, are easily related by the following proposition.

Proposition 3.5. In the fiber $\operatorname{dim}^{-1}(\mathrm{~A})$ the orders $<$ and $\tilde{<}$ are the same if $\chi(A)>0$ or reversed if $\chi(A)<0$.

ProOF. Let $a_{1}, a_{2}$ be quantities in the fiber $\operatorname{dim}^{-1}(\mathrm{~A})$ such that $a_{1}<a_{2}$ with respect to a system of units $\sigma$, that is $a_{1}=$ $\alpha_{1} \sigma(\mathrm{A})$ and $a_{2}=\alpha_{2} \sigma(\mathrm{A})$ with $\alpha_{1}, \alpha_{2} \in F$ and $\alpha_{1}<\alpha_{2}$ in $F$. With respect to another system of units $\tilde{\sigma}$ we have $a_{1}=$ $\alpha_{1} \chi(\mathrm{A})^{-1} \tilde{\sigma}(\mathrm{A})$ and $a_{2}=\alpha_{2} \chi(\mathrm{A})^{-1} \tilde{\sigma}(\mathrm{A})$. Now, if $\chi(\mathrm{A})>0$ so is $\chi^{-1}(\mathrm{~A})$ and, thus, $\alpha_{1} \chi(\mathrm{A})^{-1}<\alpha_{2} \chi(\mathrm{A})^{-1}$, so $a_{1} \tilde{<} a_{2}$, while if $\chi(\mathrm{A})<0$ the opposite is the case and $a_{2} \tilde{<} a_{1}$.

Let us denote a scale factor map $\chi: \mathscr{D} \rightarrow F^{*}$ positive if $\chi(\mathscr{D}) \subset F^{+}$, and let us denote by $\mathscr{G}^{+}$the subset of all such maps in $\mathscr{G}$. A positive map, thus, is a change of scale which does not change the orientation of any fiber, so it does not alter the order structure. The set $\mathscr{G}^{+}$is a subgroup of $\mathscr{G}$ and the quotient group encloses the freedom in the ordering possibilities for the space of quantities as stated in the following proposition.

Proposition 3.6. Let $Q \stackrel{\operatorname{dim}}{\longrightarrow} \mathscr{D}$ be a space of quantities of rank $n$ over the ordered field $F$, let $\mathscr{G}$ be the group of scale factors maps and $\mathscr{G}^{+}$the subset of positive maps. Then

i. $\mathscr{G} \cong F^{*} \times \cdots \times F^{*}(n$ copies $)$,

ii. $\mathscr{G}^{+}$is a subgroup of $\mathscr{G}$,

iii. $\mathscr{G}^{+} \cong F^{+} \times \cdots \times F^{+}($n copies $)$,

iv. $G / G^{+} \cong C_{2} \times \cdots \times C_{2}(n$ copies $)$.

PROOF. An element $\chi$ in the group $\mathscr{G}$ is determined by $n$ nonzero numbers $\left(\alpha_{1}, \ldots, \alpha_{n}\right)$ in $F$ for, given a basis $\left\{\mathrm{A}_{1}, \ldots, \mathrm{A}_{n}\right\}$ of the dimension group $\mathscr{D}$, the rule $\chi\left(\mathrm{A}_{i}\right)=\alpha_{i}$, $i=1, \ldots, n$, defines $\chi$ completely and viceversa, which proves item (i).

The identity of the group $\mathscr{G}$ is clearly a positive map and, if $\chi$ and $\xi$ are positive maps, so is its product for $(\chi \xi)(\mathrm{A})=$ $\chi(\mathrm{A}) \xi(\mathrm{A})>0$ for any $\mathrm{A} \in \mathscr{D}$, so $\mathscr{G}^{+}$is a subgroup.

The same argument of the first item applies to prove that $\mathscr{G}^{+}$is isomorphic with the direct product of $n$ copies of the positive elements of $F^{*}$.

Finally, $\mathscr{G} / \mathscr{G}^{+} \cong\left(F^{*}\right)^{n} /\left(F^{+}\right)^{n} \cong C_{2} \times \cdots \times C_{2}$ or in other words, $\mathscr{G} / \mathscr{G}^{+}$is an elementary Abelian 2-group.

The order of the quotient group is, thus, $\left|\mathscr{G} / \mathscr{G}^{+}\right|=2^{n}$, which is the freedom in choosing orientations of the fibers (we have the freedom to choose the orientation of $n$ fibers, those of a basis in $\mathscr{D}: n$ choices of two orientations gives $2^{n}$ total choices).

Notice that this freedom cannot, and must not, be reduced, for it is necessary in the actual application of quantity calculus. Perhaps the clearest example of an arbitrary choice of orientation is that of electric charge (equivalently, electric 
current, as in the SI). It is completely arbitrary that the charge of the proton has been chosen to be positive and the charge of the electron to be negative, and the physics involved do not change if we switch the signs of the charges.

\section{Dimensional Analysis}

In this section we assume the base field $F$ to be $\mathbb{R}$, the set of real numbers.

Dimensional analysis is traditionally rooted in two results known as Bridgman's theorem [10] and Buckingham's Pi-theorem [11]. However, the result of Bridgman is unnecessary in the present context, let us explain why. In his book, Bridgman distinguishes between primary and secondary quantities. The former are those which can be directly measured, such as length or time. The later are those computed from primary quantities, for instance velocity. The result of Bridgman states that, if $q_{1}, \ldots, q_{n}$ are primary quantities, any secondary quantity obtained from them has the form $\alpha q_{1}^{r_{1}} \cdots q_{n}^{r_{n}}$, where $\alpha, r_{1}, \ldots, r_{n}$ are real numbers (as in (1)). This is another reason for the general acceptance of the so far established model of quantity calculus discussed in the introduction. Notwithstanding, in the context of a space of quantities as understood in this paper this is not necessary because it is included in the axioms of the algebraic structure. Moreover, in that definition the only operations considered between quantities are product, product by a scalar, and addition of quantities of the same kind. Therefore, an expression like that of Bridgman is not permitted unless the exponents of the quantities are integer numbers. It is a matter of fact that equations of Physics involve only expressions of the form $\alpha q_{1}^{m_{1}} \cdots q_{n}^{m_{n}}$, where $\alpha \in \mathbb{R}$ but $m_{1}, \ldots, m_{n} \in \mathbb{Z}$. Indeed, the description of a theory of quantity calculus with integer exponents instead of real ones is one of the leitmotifs of the present paper and its predecessor [1].

Therefore, we are left only with the task of stating and proving the Pi-theorem in the context of the present algebra structure of quantity calculus. The first step towards such a theorem is the concept of dimensionally homogeneous equation, which seems to have been conceived firstly by Fourier [12] and succesfully employed by Reynolds [13] or Rayleigh [14]. The naive idea is that all the terms in a valid equation of Physics must have the same dimensions. Another approach says that such an equation, which describes a law of nature, must be invariant under changes in the system of units, which is arbitrary. We take the later path to define the concept of dimensional homogeneity, that is, we consider homogeneity as a kind of symmetry for it states an invariance property under suitable transformations.

The mathematical tool for dealing with symmetries is the group theory. In sections 2 and 3 a change of units has been characterized by a group homomorphism $\chi: \mathscr{D} \rightarrow F^{*}$, called a scale factor map, and the set of all those maps are gathered together in the group $\mathscr{G}$. In a fiber $\operatorname{dim}^{-1}(\mathrm{~A})$, the new unit is obtained from the previous by multiplication by the factor $\chi(\mathrm{A})$, while the numerical values of quantities get multiplied by $\chi(\mathrm{A})^{-1}$; the quantities themselves are not modified. The same numerical values are obtained if we multiply all the quantities in the same fiber by the factor $\chi(\mathrm{A})^{-1}$ and refer them to the same original unit, so we take advantage of the later to define an action of the group $\mathscr{G}$ on the space of quantities $Q$. For a quantity $q$, denote by $\chi[q]$ the action of $\chi \in \mathscr{G}$ on $q$, defined as $\chi[q]=\chi(\operatorname{dim}(q))^{-1} q$. It is an action for the identity in $\mathscr{G}$ given by $1 \mathscr{G}[q]=q$ and, given two maps $\chi, \xi \in \mathscr{G}$ we have

$$
\begin{array}{r}
\chi[\xi[q]]=\chi\left(\operatorname{dim}\left(\xi(\operatorname{dim}(q))^{-1} q\right)\right)^{-1} \xi(\operatorname{dim}(q))^{-1} q= \\
\chi(\operatorname{dim}(q))^{-1} \xi(\operatorname{dim}(q))^{-1} q=(\chi \xi)[q],
\end{array}
$$

because $\operatorname{dim}\left(\xi(\operatorname{dim}(q))^{-1} q\right)=\operatorname{dim}(q)$. Now assume the quantities $a_{1}, \ldots a_{l}, b$, with dimensions $\operatorname{dim}\left(a_{i}\right)=\mathrm{A}_{i}, \quad i=$ $1, \ldots, l, \operatorname{dim}(b)=\mathrm{B}$, are related by a formula such as $f\left(a_{1}, \ldots, a_{l}\right)=b$. We define the concept of homogeneity in terms of the map $f$ and the action of $\mathscr{G}$ just introduced..

Definition 4.1. Let $\mathrm{A}_{1}, \ldots, \mathrm{A}_{l}, \mathrm{~B}$ be dimensions in $\mathscr{D}$. A map $f: \operatorname{dim}^{-1}(\mathrm{~A}) \times \cdots \times \operatorname{dim}^{-1}\left(\mathrm{~A}_{l}\right) \rightarrow \operatorname{dim}^{-1}(\mathrm{~B})$ is dimensionally homogeneous if it commutes with the action of the group $\mathscr{G}$ in the space of quantities. That is, for any $\chi \in \mathscr{G}$ and any $a_{i} \in \mathrm{A}_{i}$, $i=1, \ldots, l$,

$$
f\left(\chi\left[a_{1}\right], \ldots, \chi\left[a_{l}\right]\right)=\chi\left[f\left(a_{1}, \ldots, a_{l}\right)\right] .
$$

Lemma 4.2. Let $f: \operatorname{dim}^{-1}(\mathrm{~A}) \times \cdots \times \operatorname{dim}^{-1}\left(\mathrm{~A}_{l}\right) \rightarrow$ $\operatorname{dim}^{-1}(\mathrm{~B})$ be a homogeneous nonzero map and let $\left\{\mathrm{A}_{1}, \ldots, \mathrm{A}_{k}\right\}$, after reordering if necessary, be a maximal independent subset of $\left\{\mathrm{A}_{1}, \ldots, \mathrm{A}_{l}\right\}$. Then the set $\left\{\mathrm{A}_{1}, \ldots, \mathrm{A}_{k}, \mathrm{~B}\right\}$ is not independent in $\mathscr{D}$, that is, there are integer numbers $n, n_{1}, \ldots, n_{k}$ such that

$$
\mathrm{B}^{n}=\mathrm{A}_{1}^{n_{1}} \cdots \mathrm{A}_{k}^{n_{k}}
$$

Proof. Assume the contrary, i.e. $\left\{A_{1}, \ldots, A_{k}, B\right\}$ is an independent set in $\mathscr{D}$. Let $a_{1}, \ldots, a_{l}, b$ be quantities of the appropriate dimensions: $\operatorname{dim}\left(a_{i}\right)=\mathrm{A}_{i}, i=1, \ldots, l, \operatorname{dim}(b)=\mathrm{B}$, such that $b=f\left(a_{1}, \ldots, a_{l}\right) \neq 0$. By the assumption we can choose arbitrary scalars in $F^{*}, \alpha_{1}, \ldots, \alpha_{k}, \beta$ and find a group homomorphism $\chi: \mathscr{D} \rightarrow F^{*}$ such that $\chi\left(\mathrm{A}_{i}\right)=\alpha_{i}, i=1, \ldots, k$ and $\chi(\mathrm{B})=\beta$. Take, for instance, $\alpha_{1}=\cdots=\alpha_{k}=1, \beta=2$. Then the action of $\chi$ on the quantities involved is $\chi\left[a_{i}\right]=a_{i}$, $i=1, \ldots, l$ (for the cases $i>k$, there is a relationship between $A_{i}$ and $A_{1}, \ldots, A_{k}$, so we can choose $\chi\left(A_{i}\right)=1$ as well), $\chi[b]=\frac{1}{2} b$. Now, since the map $f$ is homogeneous we find

$$
\begin{aligned}
b=f\left(a_{1}, \ldots, a_{l}\right)=f\left(\chi\left[a_{1}\right], \ldots,\right. & \left.\chi\left[a_{l}\right]\right) \\
& =\chi\left[f\left(a_{1}, \ldots, a_{l}\right)\right]=\frac{1}{2} b
\end{aligned}
$$

a contradiction.

We also need a lemma on the extraction of $n$th roots of suitable quantities.

Lemma 4.3. Let $Q \stackrel{\operatorname{dim}}{\longrightarrow} \mathscr{D}$ be an ordered space of quantities over $\mathbb{R}$ oriented by a system of units $\sigma$, let $A \in \mathscr{D}$ be a dimension and $q$ a quantity with $\operatorname{dim}(q)=\mathrm{A}^{n}, n$ an integer number. 
i. If $n$ is an odd integer there exists a quantity $a$, with $\operatorname{dim}(a)=\mathrm{A}$, such that $a^{n}=q$.

ii. If $n$ is an even integer and $q$ is positive there exists $a$ quantity $a$, with $\operatorname{dim}(a)=\mathrm{A}$, such that $a^{n}=q$.

Proof. The hypothesis on $q$ allows us to write $q=\beta \sigma\left(\mathrm{A}^{n}\right)$, where $\beta \in \mathbb{R}$ and, in case (ii), $\beta>0$. In both cases, there exists in $\mathbb{R}$ the $n$th root of this number, say $\beta^{\frac{1}{n}}$, and we just define $a=\beta^{\frac{1}{n}} \sigma(\mathrm{A})$.

The point in the previous lemma is to understand to what extent the condition on $q$ being positive in case (ii) is not an empty one, since the orientation of fibers is dependent on the system of units, which is arbitrary. However, the orientation of the fiber with dimension $\mathrm{A}^{n}$ is not arbitrary when $n$ is even. Consider two systems of units, $\sigma$ and $\tilde{\sigma}$ which give different orientations to the fiber with dimension $A$, say $\tilde{\sigma}(\mathrm{A})=-\sigma(\mathrm{A})$. Then $\tilde{\sigma}\left(\mathrm{A}^{n}\right)=\tilde{\sigma}(\mathrm{A})^{n}=(-\sigma(\mathrm{A}))^{n}=\sigma\left(\mathrm{A}^{n}\right)$ so the fiber $\operatorname{dim}^{-1}\left(A^{n}\right)$ has only one possible orientation.

We are now ready to state and prove the Pi-theorem.

Theorem 4.4 (Pi-theorem with integer exponents). Let $Q \stackrel{\mathrm{dim}}{\longrightarrow}$ $\mathscr{D}$ be an ordered space of quantities over the field $\mathbb{R}$ oriented by a system of units. Let $\mathrm{A}_{1}, \ldots, \mathrm{A}_{l}, \mathrm{~B} \in \mathscr{D}$ be dimensions and $\left\{A_{1}, \ldots, A_{k}\right\}$, after reordering if necessary, be a maximal independent subset of $\left\{\mathrm{A}_{1}, \ldots, \mathrm{A}_{l}\right\}$.

If $f: \operatorname{dim}^{-1}\left(\mathrm{~A}_{1}\right) \times \cdots \times \operatorname{dim}^{-1}\left(\mathrm{~A}_{l}\right) \rightarrow \operatorname{dim}^{-1}(\mathrm{~B})$ is a homogeneous map and $a_{1}, \ldots, a_{l}$ are positive quantities in their respective fibers, $\operatorname{dim}\left(a_{i}\right)=\mathrm{A}_{i}, i=1, \ldots, l$, then there are integer numbers $n, n_{1}, \ldots, n_{k}$ and a function $g: \mathbb{R}^{l-k} \rightarrow \mathbb{R}$ such that

$$
f\left(a_{1}, \ldots, a_{l}\right)=\left(a_{1}^{n_{1}} \cdots a_{k}^{n_{k}}\right)^{\frac{1}{n}} \quad g\left(\pi_{k+1}, \ldots, \pi_{l}\right),
$$

for suitable real numbers $\pi_{k+1}, \ldots, \pi_{l}$.

The real numbers $\pi_{k+1}, \ldots, \pi_{l}$ are the $l-k$ independent dimensionless quantities that can be constructed with the original quantities $a_{1}, \ldots, a_{l}$ (the so called pi-groups which give the name to the theorem).

Proof. By the hypothesis for each $A_{j}$ not in the subset $\left\{\mathrm{A}_{1}, \ldots, \mathrm{A}_{k}\right\}$ there are integer numbers $m_{j}, m_{j 1}, \ldots, m_{j k}$ such that

$$
\mathrm{A}_{j}^{m_{j}}=\mathrm{A}_{1}^{m_{j 1}} \cdots \mathrm{A}_{k}^{m_{j k}}, \quad k \leq j \leq l .
$$

By lemma 4.2 there are integer numbers $n, n_{1}, \ldots, n_{k}$ such that

$$
\mathrm{B}^{n}=\mathrm{A}_{1}^{n_{1}} \cdots \mathrm{A}_{k}^{n_{k}} .
$$

For each $j=k+1, \ldots, l$ define

$$
\pi_{j}=a_{j}\left(a_{1}^{-m_{j 1}} \cdots a_{k}^{-m_{j k}}\right)^{\frac{1}{m_{j}}},
$$

which is well defined because, by (8), the quantity $a^{-m_{j 1}} \ldots a_{k_{j}}^{-m_{j k}}$ has dimension $\mathrm{A}_{j}^{-m_{j}}$ and so the $m_{j}$ th root does exist. The dimension of $\pi_{j}$ can be thus computed as

$$
\begin{array}{r}
\operatorname{dim}\left(\pi_{j}\right)=\operatorname{dim}\left(a_{j}\right) \operatorname{dim}\left(\left(a_{1}^{-m_{j 1}} \cdots a_{k}^{-m_{j k}}\right)^{\frac{1}{m_{j}}}\right) \\
=\mathrm{A}_{j} \mathrm{~A}_{j}^{-1}=1_{\mathscr{D}},
\end{array}
$$

so it is dimensionless.

Now, in order to take advantage of the homogeneity of $f$ we define a suitable scale factor map $\chi: \mathscr{D} \rightarrow \mathbb{R}^{*}$. Since $\left\{\mathrm{A}_{1}, \ldots, \mathrm{A}_{k}\right\}$ is independent in $\mathscr{D}$ we can certainly choose arbitrarily $k$ nonzero real numbers as image of $\chi\left(\mathrm{A}_{i}\right), i=$ $1, \ldots, k$. Let us take $\chi\left(\mathrm{A}_{i}\right)=v\left(a_{i}\right), i=1, \ldots, k$, where $v$ is the map defined in (4). Then the action of $\chi$ is $\chi\left[a_{i}\right]=$ $v\left(a_{i}\right)^{-1} a_{i}=\sigma\left(\mathrm{A}_{i}\right)$, the unit in the fiber, for $i=1, \ldots, k$. However, for $j=k+1, \ldots, l$ the result is different, for

$$
\begin{aligned}
& \chi\left(\mathrm{A}_{j}\right)^{m_{j}}=\chi\left(\mathrm{A}_{j}^{m_{j}}\right)= \\
& \chi\left(\mathrm{A}_{1}^{m_{j 1}} \cdots \mathrm{A}_{k}^{m_{j k}}\right)=\chi\left(\mathrm{A}_{1}\right)^{m_{j 1}} \cdots \chi\left(\mathrm{A}_{k}\right)^{m_{j k}}= \\
& \quad v\left(a_{1}\right)^{m_{j 1}} \cdots v\left(a_{k}\right)^{m_{j k}}=v\left(a_{1}^{m_{j 1}} \cdots a_{k}^{m_{j k}}\right)
\end{aligned}
$$

since both, $\chi$ and $v$, are homomorphisms.

From (10) we have $a_{j}^{m_{j}}=\pi_{j}^{m_{j}} a_{1}^{m_{j 1}} \cdots a_{k}^{m_{j k}}$. Therefore $\chi\left(\mathrm{A}_{j}\right)^{m_{j}}=v\left(\pi_{j}^{-m_{j}} a_{j}^{m j}\right)=\pi_{j}^{-m_{j}} v\left(a_{j}^{m j}\right)$ which is a positive real number, so we conclude

$$
\chi\left(\mathrm{A}_{j}\right)=\pi_{j}^{-1} v\left(a_{j}\right), \quad j=k+1, \ldots, l
$$

and the action is

$$
\chi\left[a_{j}\right]=\pi_{j} v\left(a_{j}\right)^{-1} a_{j}=\pi_{j} \sigma\left(\mathrm{A}_{j}\right), \quad j=k+1, \ldots, l .
$$

With a parallel argument we arrive at

$$
\chi(B)=\left(v\left(a_{1}\right)^{n_{1}} \cdots v\left(a_{k}\right)^{n_{k}}\right)^{\frac{1}{n}},
$$

so the action on $b$ is

$$
\chi[b]=\left(v\left(a_{1}\right)^{n_{1}} \cdots v\left(a_{k}\right)^{n_{k}}\right)^{\frac{-1}{n}} b
$$

which is equal, by the homogeneity of $f$, to

$$
\begin{aligned}
& f\left(\chi\left[a_{1}\right], \ldots, \chi\left[a_{k}\right], \chi\left[a_{k+1}\right], \ldots, \chi\left[a_{l}\right]\right) \\
& =f\left(\sigma\left(\mathrm{A}_{1}\right), \ldots, \sigma\left(\mathrm{A}_{k}\right), \pi_{k+1} \sigma\left(\mathrm{A}_{k+1}\right), \ldots, \pi_{l} \sigma\left(\mathrm{A}_{l}\right)\right) .
\end{aligned}
$$

Define the real function $g: \mathbb{R}^{l-k} \rightarrow \mathbb{R}$ by

$$
\begin{aligned}
& g\left(\pi_{k+1}, \ldots, \pi_{l}\right) \\
= & \sigma(B)^{-1} f\left(\sigma\left(\mathrm{A}_{1}\right), \ldots, \sigma\left(\mathrm{A}_{k}\right), \pi_{k+1} \sigma\left(\mathrm{A}_{k+1}\right), \ldots, \pi_{l} \sigma\left(\mathrm{A}_{l}\right)\right) .
\end{aligned}
$$

The right hand side of (16) lies in the fiber of dimensionless quantities which, once again, is identified with the base field $\mathbb{R}$. Now, by (14) and (15) and bringing together (9) and (16) we find

$$
\begin{gathered}
f\left(a_{1}, \ldots, a_{l}\right)=b= \\
\quad\left(v\left(a_{1}\right)^{n_{1}} \ldots v\left(a_{k}\right)^{n_{k}}\right)^{\frac{1}{n}} \times \\
f\left(\sigma\left(\mathrm{A}_{1}\right), \ldots, \sigma\left(\mathrm{A}_{k}\right), \pi_{k+1} \sigma\left(\mathrm{A}_{k+1}\right), \ldots, \pi_{l} \sigma\left(\mathrm{A}_{l}\right)\right)= \\
\left(v\left(a_{1}\right)^{n_{1}} \cdots v\left(a_{k}\right)^{n_{k}}\right)^{\frac{1}{n}} \sigma(\mathrm{B}) g\left(\pi_{k+1}, \ldots, \pi_{l}\right)= \\
\left(a_{1}^{n_{1}} \cdots a_{k}^{n_{k}}\right)^{\frac{1}{n}} g\left(\pi_{k+1}, \ldots, \pi_{l}\right) .
\end{gathered}
$$




\section{DifFERENTIAL AND INTEGRAL CALCULUS}

Differential and integral calculus are, possibly, the most important mathematical tools in Physics. Therefore, their use with quantities has to be justified in the context of a space of quantities. The first step, however, is to provide the space of quantities with a topology so that the concept of limit can be properly handled. Since all these concepts make sense in a complete field and we want also to preserve the order structure, we need a complete ordered field as base field, that is, the field of real numbers so, in this section, it is also assumed $F=\mathbb{R}$.

Since the definition of a space of quantities $Q \stackrel{\operatorname{dim}}{\longrightarrow} \mathscr{D}$ is purely algebraic, the only hint to impose a topology on it is that the operations in $Q$ be continuous maps, as well as the projection map, dim, on the group of dimensions $\mathscr{D}$.

We also want to take advantage of the order structure defined in section 3. Remember that the standard topology of $\mathbb{R}$ can be thought of as induced by its order, so the same can be applied to each fiber. With this topology the operations of addition and product by scalar numbers are both continuous as they are in $\mathbb{R}$.

On the other hand, the fibers which make the space of quantities are bound by the algebraic properties which are summarized in the group structure of the group of dimensions $\mathscr{D}$. This is, by definition, a finitely generated free Abelian group. There are several topologies that can be assumed in the group $\mathscr{D}$ which make it into a topological group, but we choose to endow it with the discrete topology because, first, it is the choice coherent with our previous choice of discarding an order relation among quantities of different dimensions and, second, it enables us to state and prove proposition 5.1 which is quite a natural result to be expected.

Finally, in [1] it is shown that a space of quantities $Q \stackrel{\mathrm{dim}}{\longrightarrow}$ $\mathscr{D}$ over the field $\mathbb{R}$ is algebraically isomorphic with $\mathscr{D} \times \mathbb{R}$ with suitable operations defined therein. This is to say, $Q$ can be seen as a trivial fiber bundle with base $\mathscr{D}$ and fiber $\mathbb{R}$. Therefore, it is only natural to assume as topology for $Q$ the product topology in $\mathscr{D} \times \mathbb{R}$. Accordingly to the previous two paragraphs, a base for this topology is that of open intervals in each fiber. The map $\operatorname{dim}: Q \rightarrow \mathscr{D}$ is trivially continuous. Thus, the space of quantities can be described in this respect as a bundle whose fibers are 1-dimensional positively oriented linearly ordered order-topological vector spaces.

Now we consider quantities as variables in order to define maps and deal with them towards the definition of derivative. A variable quantity often depends on a real parameter, so we consider first this case and study the conditions for such a map to be continuous. The first proposition shows that a continuous map in $Q$ must lie on a single fiber.

Proposition 5.1. Let $f: \mathbb{R} \rightarrow Q$ be continuous. Then $f(\mathbb{R})$ lies in a single fiber of $Q$.

PROOF. Let us prove the equivalent statement: if $f(\mathbb{R})$ is not contained in a single fiber, then $f$ is not continuous. Let $x, y$ be real numbers such that $\operatorname{dim}(f(x)) \neq \operatorname{dim}(f(y))$ and assume $x<y$. Define the subset of $\mathbb{R}$ of the numbers less than $y$ and with image by $f$ in the same fiber that $x$,
$S=\{z \in \mathbb{R}: \operatorname{dim}(f(z))=\operatorname{dim}(f(x))$ and $z<y\}$, which is a nonempty set $(x \in S)$ and with upper bound $y$. Denote $y_{0}$ the least upper bound of $S$, which is an accumulation point of $S$, and consider the following two possible choices.

First, if $y_{0} \in S$, then $y_{0}<y$ and $\operatorname{dim}\left(f\left(y_{0}\right)\right)=\operatorname{dim}(f(x))=$ A. We now show that the inverse image of the set $\{A\}$, which is open in $\mathscr{D}$, by the map $\operatorname{dim} \circ f$ is not open in $Q$, for $y_{0}$ is in that inverse image but it is not an interior point. In fact, any open ball in the fiber centered on $y_{0}$ and radius less than $\left|y-y_{0}\right|$ contains points not in $S$ and not in $(\operatorname{dim} \circ f)^{-1}(\mathrm{~A})$. Therefore, $\operatorname{dim} \circ f$ is not a continuous map, and so is $f$ because dim is continuous.

Second, if $y_{0} \notin S$, apply the same argument to the complementary set of $\{A\}$ in $\mathscr{D}$, which is again an open set. Its inverse image by $\operatorname{dim} \circ f$ contains $y_{0}$ which, again, is not an interior point.

Therefore, a necessary condition for a map $f: \mathbb{R} \rightarrow Q$ to be continuous is that $f(\mathbb{R})$ is contained in a single fiber in $Q$.

It is also usual to consider a variable quantity as a parameter for another, e.g. time as parameter for the length travelled by a moving particle. Since any single fiber is homeomorphic with $\mathbb{R}$, the previous proposition applies to this case as well. It is stated in the following corollary.

Corollary 5.2. Let $\mathrm{A}$ be a dimension in $\mathscr{D}$ and $\operatorname{dim}^{-1}(\mathrm{~A})$ its fiber in $Q$. If a map $f: \operatorname{dim}^{-1}(\mathrm{~A}) \rightarrow Q$ is continuous, then its image is contained in a single fiber of $Q$.

In the following, we only consider maps of the form $f$ : $\mathbb{R} \rightarrow \operatorname{dim}^{-1}(\mathrm{~B})$ or $f: \operatorname{dim}^{-1}(\mathrm{~A}) \rightarrow \operatorname{dim}^{-1}(\mathrm{~B})$, which include all continuous maps, so in fact we are considering maps of the form $\mathbb{R} \rightarrow \mathbb{R}$ and, thus, differential and integral calculus can be readily established. But before we give a definition of derivative and of integral with quantities it proves useful to point how limits of functions can be dealt with in the fashion of metric spaces. Once we have adopted a topology in $Q$ the concept of limit of a map in $Q$ is established. Nevertheless, the following lemma states that the definition of limit in $Q$ can be reduced to that in $\mathbb{R}$ due to that topology.

Lemma 5.3. Let $\mathrm{A}, \mathrm{B}$ be dimensions in $\mathscr{D}, a, b$ quantities with $\operatorname{dim}(a)=\mathrm{A}, \operatorname{dim}(b)=\mathrm{B}$, and $f: \operatorname{dim}^{-1}(\mathrm{~A}) \rightarrow \operatorname{dim}^{-1}(\mathrm{~B}) a$ map. The limit of $f$ at $a$ is $b$ iffor any positive quantity $\varepsilon$ with $\operatorname{dim}(\varepsilon)=\mathrm{B}$, there is a positive quantity $\delta$ with $\operatorname{dim}(\delta)=\mathrm{A}$, such that, given $a^{\prime} \in \operatorname{dim}^{-1}(\mathrm{~A})$, if $\left|a^{\prime}-a\right|<\delta$ then $\mid f\left(a^{\prime}\right)-$ $b \mid<\varepsilon$.

Proof. A basis for the topology in $Q$ is obtained from the product of a basis in $\mathscr{D}$ (single elements) and a basis in $\mathbb{R}$ (open intervals or balls); we get, thus, open intervals in each fiber. Now apply the standard definition of limit in a given topology, but take into account that any open set containing $a$ can be substituted by an open ball in the fiber $\operatorname{dim}^{-1}(A)$ centered in $a$, and the same applies for $b$. An open ball centered on $a$ is described a $\left|a^{\prime}-a\right|<\delta$, while the open ball centered on $b$ can be described as $\left|b^{\prime}-b\right|<\varepsilon$, so the lemma follows.

For the sake of completeness the definitions of the derivative and of the integral follow. 
Definition 5.4. Let $Q \stackrel{\mathrm{dim}}{\longrightarrow} \mathscr{D}$ be a space of quantities over the field $\mathbb{R}$, let $\mathrm{A}, \mathrm{B}$ be dimensions in $\mathscr{D}$ and let $f: \operatorname{dim}^{-1}(\mathrm{~A}) \rightarrow$ $\operatorname{dim}^{-1}(\mathrm{~B})$ be a map. The map $f$ is differentiable at $a \in$ $\operatorname{dim}^{-1}(A)$ if the following limit exists

$$
\lim _{h \rightarrow 0_{\mathrm{A}}} \frac{f(a+h)-f(a)}{h},
$$

where $\operatorname{dim}(h)=\mathrm{A}$. In such case, the value of the limit is denoted $f^{\prime}(a)$ and referred to as the derivative of $f$ at a.

The definition shows, in addition, that the dimension of the derivative is that of the image of the map times the inverse of the independent variable quantity:

$$
\operatorname{dim}\left(f^{\prime}(a)\right)=\operatorname{dim}(f(a)) \operatorname{dim}(a)^{-1}=\mathrm{BA}^{-1} .
$$

Starting from this definition the rest of the differential calculus in $\mathbb{R}$ can be traslated step by step to $Q$ straighforward, so we will not go further into it here.

Now we turn to the definition of the integral of a map. Since the goal is to traslate the concepts defined in $\mathbb{R}$ to a space of quantities, the simpler case of Riemannian definition of integral suffices to our purposes.

Let $\left[a^{\prime}, a^{\prime \prime}\right]$ be an interval in the fiber $\operatorname{dim}^{-1}(\mathrm{~A})$ and let $P=$ $\left\{a_{0}, a_{1}, \ldots, a_{n}\right\}$, where $a^{\prime}=a_{0}<a_{1}<\cdots<a_{n}=a^{\prime \prime}$, be a partition of that interval. Let $f: \operatorname{dim}^{-1}(\mathrm{~A}) \rightarrow \operatorname{dim}^{-1}(\mathrm{~B})$ be a map bounded in $\left[a^{\prime}, a^{\prime \prime}\right]$. For each $i=1, \ldots, n$ define

$$
\begin{aligned}
& m_{i}=\inf \left\{f(a): a_{i-1} \leq a \leq a_{i}\right\}, \\
& M_{i}=\sup \left\{f(a): a_{i-1} \leq a \leq a_{i}\right\} .
\end{aligned}
$$

The lower and upper sums of $f$ for the partition $P$ are, respectively,

$$
\begin{array}{r}
L(f, P)=\sum_{i=1}^{n} m_{i}\left(a_{i}-a_{i-1}\right), \\
U(f, P)=\sum_{i=1}^{n} M_{i}\left(a_{i}-a_{i-1}\right) .
\end{array}
$$

Then the properties regarding lower and upper sums with different partitions can be readily proven (see, e.g. [15]) and the following definition stated

Definition 5.5. Let $Q \stackrel{\mathrm{dim}}{\longrightarrow} \mathscr{D}$ be a space of quantities over the field $\mathbb{R}$, let $\mathrm{A}, \mathrm{B}$ be dimensions in $\mathscr{D}$ and let $f: \operatorname{dim}^{-1}(\mathrm{~A}) \rightarrow$ $\operatorname{dim}^{-1}(\mathrm{~B})$ be a bounded map in the interval $\left[a^{\prime}, a^{\prime \prime}\right] \subset$ $\operatorname{dim}^{-1}(\mathrm{~A})$. The map $f$ is integrable in that interval if

$$
\begin{aligned}
& \sup \left\{L(f, P): P \text { a partition of }\left[a^{\prime}, a^{\prime \prime}\right]\right\}= \\
& \quad \inf \left\{U(f, P): \text { P a partition of }\left[a^{\prime}, a^{\prime \prime}\right]\right\} .
\end{aligned}
$$

In such a case, this number is denoted $\int_{a^{\prime}}^{a^{\prime \prime}} f(a) \mathrm{d} a$ and referred to as the integral of $f$ in the interval $\left[a^{\prime}, a^{\prime \prime}\right]$.

The definition and (18) show that the dimension of the integral is

$$
\begin{aligned}
& \operatorname{dim}\left(\int_{a^{\prime}}^{a^{\prime \prime}} f(a) \mathrm{d} a\right)=\operatorname{dim}\left(\sum m_{i}\left(a_{i}-a_{i-1}\right)\right)= \\
& \operatorname{dim}(f(a)) \operatorname{dim}(a)=\mathrm{BA} .
\end{aligned}
$$

\section{ACKNOWLEDGEMENT}

Thanks are due to the anonymous reviewers whose insightful and accurate comments have improved this paper.

\section{REFERENCES}

[1] Raposo, A. P. (2018). The algebraic structure of quantity calculus. Measurement Science Review 18(4), 147-157.

[2] JCGM (2012). International vocabulary of metrologybasic and general concepts and associated terms (vim). 3rd edition.

[3] Kitano, M. (2013). Mathematical structure of unit systems. Journal of Mathematical Physics 54, 052901-117.

[4] Drobot, S. (1953). On the foundations of dimensional analysis. Studia Mathematica 14 (1), 84-99.

[5] Whitney, H. (1968). The mathematics of physical quantities: Part II: Quantity structures and dimensional analysis. The American Mathematical Monthly 75, 227256.

[6] Carlson, D. (1979). A mathematical theory of physical units, dimensions and measures. Archive for Rational Mechanics and Analysis 70 (4), 289-304.

[7] Maxwell, J. (1873). Treatise on Electricity and Magnetism. Oxford University Press.

[8] de Boer, J. (1994). On the history of quantity calculus and the international system. Metrologia 31, 405-429.

[9] Krystek, M. (2015). The term 'dimension' in the international system of units. Metrologia 52, 297-300.

[10] Bridgman, P. Dimensional Analysis. Yale University Press.

[11] Buckingham, E. (1914). On physically similar systems: Illustrations of the use of dimensional equations. Physical Review 4, 345-376.

[12] Fourier, J. (1822). Théorie analytique de la chaleur. Chez Firmin Didot, père et fils.

[13] Reynolds, O. (1883). An experimental investigation of the circumstances which determine whether the motion of water shall be direct or sinuous, and of the law of resistance in parallel channels. Philosophical transactions of the Royal Society of London 174, 935-982.

[14] Rayleigh, L. (1892). On the question of the stability of the flow of fluids. The London, Edinburgh, and Dublin Philosophical Magazine and Journal of Science 34(206), 59-70.

[15] Spivak, M. (1980). Calculus. Publish or Perish, 2nd edition.

Received November 15, 2018. Accepted April 10, 2019. 